\title{
Differential lineage-specific amplification of transposable elements is responsible for genome size variation in Gossypium
}

\author{
Jennifer S. Hawkins, ${ }^{1}$ HyeRan Kim, ${ }^{2}$ John D. Nason, ${ }^{1}$ Rod A. Wing, ${ }^{2}$ \\ and Jonathan F. Wendel ${ }^{1,3}$ \\ ${ }^{1}$ Iowa State University, Department of Ecology, Evolution and Organismal Biology, Ames, lowa 50011, USA; ${ }^{2}$ University \\ of Arizona, Department of Plant Sciences, Arizona Genomics Institute, Tucson, Arizona 85721, USA
}

\begin{abstract}
The DNA content of eukaryotic nuclei (C-value) varies $\sim 200,000$-fold, but there is only a $\sim 20$-fold variation in the number of protein-coding genes. Hence, most $\mathrm{C}$-value variation is ascribed to the repetitive fraction, although little is known about the evolutionary dynamics of the specific components that lead to genome size variation. To understand the modes and mechanisms that underlie variation in genome composition, we generated sequence data from whole genome shotgun (WGS) libraries for three representative diploid $(n=13)$ members of Gossypium that vary in genome size from 880 to $2460 \mathrm{Mb}(\mathrm{IC})$ and from a phylogenetic outgroup, Gossypioides kirkii, with an estimated genome size of $588 \mathrm{Mb}$. Copy number estimates including all dispersed repetitive sequences indicate that $40 \%-65 \%$ of each genome is composed of transposable elements. Inspection of individual sequence types revealed differential, lineage-specific expansion of various families of transposable elements among the different plant lineages. Copia-like retrotransposable element sequences have differentially accumulated in the Gossypium species with the smallest genome, G. raimondii, while gypsy-like sequences have proliferated in the lineages with larger genomes. Phylogenetic analyses demonstrated a pattern of lineage-specific amplification of particular subfamilies of retrotransposons within each species studied. One particular group of gypsy-like retrotransposon sequences, Gorge3 (Gossypium retrotransposable gypsy-like element), appears to have undergone a massive proliferation in two plant lineages, accounting for a major fraction of genome-size change. Like maize, Gossypium has undergone a threefold increase in genome size due to the accumulation of LTR retrotransposons over the 5-10 Myr since its origin.
\end{abstract}

[The sequence data described in this paper have been submitted to the GSS Division of GenBank under accessions DX390732-DX406528.]

Genomes of eukaryotic organisms vary over 200,000-fold in size, ranging from $2.8 \mathrm{Mb}$ in Encephalitozoon cuniculi (Biderre et al. 1998 ) to $>690,000 \mathrm{Mb}$ in the diatom Navicola pelliculosa (Cavalier-Smith 1985; Li and Graur 1991). Among angiosperms, genome sizes range from $\sim 108 \mathrm{Mb}$ for Fragaria viridis (Bennett and Leitch 2005) to $>120,000 \mathrm{Mb}$ in some members of the Liliaceae (Flavell et al. 1974; Bennett and Smith 1991; Bennett and Leitch 1995, 1997; Leitch et al. 1998). Not only is wide variation in genome size common among distantly related organisms, but it also is unexceptional even among closely related species. For example, genome sizes range approximately sixfold among members of the genus Vicia (Chooi 1971), and ninefold within the genus Crepsis (Jones and Brown 1976). Some portion of this genome size variation may be ascribed to differences in gene number amplification due to gene, chromosome segment, and wholegenome duplication, as well as to gene loss (Tikhonov et al. 1999; Blanc et al. 2000; Grant et al. 2000; Ku et al. 2000; Vision et al. 2000; Wendel 2000; Bancroft 2001; Bennetzen and Ramakrishna 2002). Nevertheless, $>90 \%$ of plant genes possess close homologs within other plant species, indicative of highly conserved gene content (Bennetzen 2000a).

${ }^{3}$ Corresponding author.

E-mail jfw@iastate.edu; fax (515) 294-1337.

Article published online before print. Article and publication date are at http:// www.genome.org/cgi/doi/10.1101/gr.5282906.
There appears to be no correlation between the amount of DNA per cell and organismal advancement or genetic complexity (Sparrow et al. 1972; Price 1988). This well-documented lack of correspondence between genome size and morphological or physiological complexity of an organism has been historically termed the "C-value paradox" (Thomas 1971). Since the discovery of non-coding DNA and its impact on genome size variation, "paradox" has been replaced by "enigma" in an attempt to more appropriately identify the topic as a "perplexing subject" made up of several independent components (Gregory 2002, 2004). It is now generally agreed that the C-value enigma can be largely explained by the differential amplification and proliferation among organisms of the repetitive fraction of the genome (Bennetzen 2000b, 2002; Kidwell 2002).

In plants, amplification and insertion of newly activated long terminal repeat (LTR) retrotransposable elements appear to be major contributors to genome size expansion. For example, $\sim 70 \%$ of the maize nuclear genome is composed of LTRretrotransposons (SanMiguel and Bennetzen 1998). In the span of just a few million years, the maize genome doubled in size due to transposable element (TE) activity (SanMiguel and Bennetzen 1998). These TEs are often found in nested arrangements located between "gene islands," and often are associated with centromeres (SanMiguel et al. 1996). To date, little is known regarding the extent to which various TEs contribute to genome size variation or how TE types are distributed among closely related spe- 
cies. Other mechanisms posited to be responsible for genome size expansion include variation in intron size (Deutsch and Long 1999), expansion of tandemly repetitive DNA sequences (Ellegren 2002; Morgante et al. 2002), segmental duplication (Blanc et al. 2000; $\mathrm{Ku}$ et al. 2000; Vision et al. 2000; Wendel 2000; Bancroft 2001), accumulation of pseudogenes (Zhang 2003), and transfer of organellar DNA to the nucleus (Adams and Palmer 2003; Shahmuradov et al. 2003). However, these mechanisms generally do not appear to have a large impact on genome size differences among closely related species.

Although it has been suggested that organisms may have a "one-way ticket to genomic obesity" (Bennetzen and Kellogg 1997), it is possible that differences in genome size are not only the outcome of an organism's tolerance for accrual of nongenic DNA, but also its efficiency in removal of non-essential DNA (Petrov and Hartl 1997; Petrov et al. 2000; Petrov 2002a; Wendel et al. 2002b). Many organisms with smaller genomes are striking in their relatively small proportion of nongenic DNA. Evidence of a deletional bias among organisms with smaller versus larger genomes (Bennett and Leitch 1997; Petrov and Hartl 1997; Kirik et al. 2000; Petrov et al. 2000) has led to the "mutational equilibrium model" of DNA loss (Petrov 2002b). Other suggested mechanisms of DNA loss include unequal intrastrand homologous recombination between two tandem repeats in the same orientation, such as the LTRs of retrotransposable elements (Shepherd et al. 1984; SanMiguel et al. 1996; Chen et al. 1998; Vicient et al. 1999; Bennetzen 2002), illegitimate recombination (Devos et al. 2002; Wicker et al. 2003; Ma et al. 2004; Bennetzen et al. 2005), and double-stranded break repair (Kirik et al. 2000; Orel and Puchta 2003; Filkowski et al. 2004).

To effectively study genome size evolution from a phylogenetic perspective, it is necessary to exploit a system in which the closely related species vary widely in genome size and for whom phylogenetic relationships are well understood. A good example in this respect is the monophyletic genus Gossypium (Malvaceae), which is composed of $\sim 50$ species of small trees and shrubs with an aggregate distribution that encompasses many tropical and subtropical semi-arid regions of the world (Fryxell 1992; Seelanan et al. 1997; Cronn et al. 2002; Wendel and Cronn 2003). Diploid members of the genus are divided into eight groups based on chromosome pairing behavior and fertility in interspecific hybrids (Beasley 1941; Endrizzi et al. 1985). All diploid members of the genus have 13 chromosomes, yet genome sizes range approximately threefold, from a median estimate of $885 \mathrm{Mb}$ per haploid nucleus in the American D-genome species, to $2572 \mathrm{Mb}$ per haploid nucleus in the Australian K-genome species (Fig. 1; Hendrix and Stewart 2005). An even larger range in genome size is observed in the tribe to which Gossypium belongs (the Gossypieae), from only $590 \mathrm{Mb}$ in Gossypioides kirkii and Kokia drynarioides to $4018 \mathrm{Mb}$ in Thespesia populnea (Wen- del et al. 2002b). The wide range in genome size observed across closely related diploid species and the well-established phylogeny makes Gossypium an excellent system for the study of genome size evolution.

To better appreciate the relevance of genome size variation to organismal fitness and evolution, it is first necessary to enhance our understanding of the quantity and quality of the genomic components that distinguish two or more genomes, as well as the modes and mechanisms by which these differences arise. This insight may derive from comparative sequence analysis of specific genomic regions or from using more global approaches. An example of the former is the recent study by Grover et al. (2004), who compared $\sim 104 \mathrm{~kb}$ of aligned sequence surrounding the CesA1 gene from the D- and A- genomes of tetraploid cotton. In this case, both gene content and intergenic regions were largely conserved, and hence there was no evidence of the mechanisms responsible for the twofold size variation that characterizes these genomes. Here, we employ the second approach, utilizing whole genome shotgun (WGS) libraries constructed for three members of Gossypium that range threefold in genome size, and one outgroup species, Gossypioidies kirkii. Copy number estimates for several Gossypium transposable elements suggest that different types of repetitive sequences have accumulated at different rates in different plant lineages. Additionally, the results suggest that different families within a repetitive sequence type proliferate differentially. Indeed, the major fraction of the genome size variation observed in Gossypium is largely due to recent, lineage-specific amplification of one particular group of gypsy-like retrotransposon sequences, Gorge3 (Gossypium retrotransposable gypsy-like element), within the larger-genome Gossypium species.

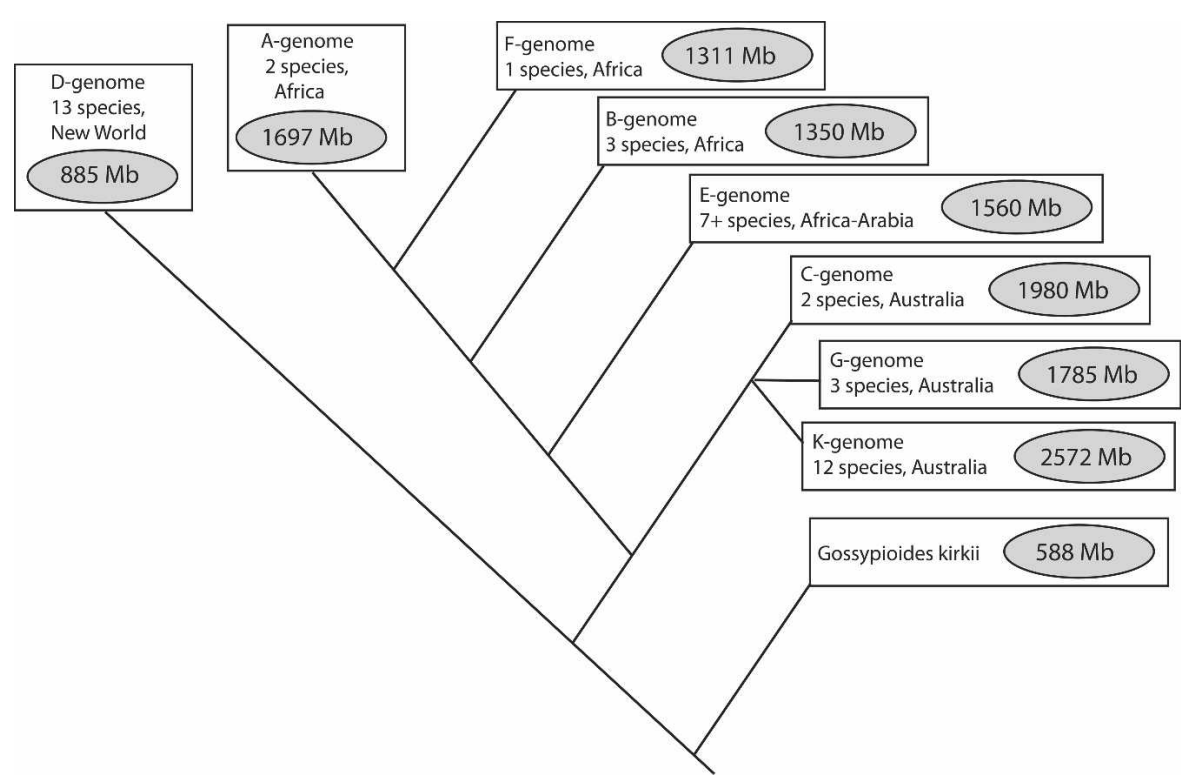

Figure 1. Evolutionary relationships among diploid members of Gossypium. Gossypium is a monophyletic genus composed of $\sim 50$ species that are widely distributed throughout many tropical and subtropical regions. Diploid species have a haploid complement of 13 chromosomes. Gossypium is divided into eight genome groups based on cytogenetic data and level of fertility in interspecific hybrids (Endrizzi et al. 1985). Multiple molecular data sets support the phylogenetic relationships indicated, including the outgroup relationship of Gossypioides kirkii (Wendel and Albert 1992; Seelanan et al. 1997; Small et al. 1998, 1999). Despite conservation of chromosome number among the diploids, genome size varies threefold, from an average of $885 \mathrm{Mb}$ in the New World D-genome species to an average of $2576 \mathrm{Mb}$ in the Australian K-genome species (Hendrix and Stewart 2005). 
Hawkins et al.

Table 1. Library construction and sequencing effort for three species representing different Gossypium genomes and one phylogenetic outgroup

\begin{tabular}{|c|c|c|c|c|c|c|}
\hline Taxon/genome group & $\begin{array}{l}\text { Genome size }{ }^{a} \\
\text { (in Mb) }\end{array}$ & $\begin{array}{l}\text { No. clones } \\
\text { in library }\end{array}$ & $\begin{array}{l}\text { Successfully } \\
\text { sequenced }\end{array}$ & $\begin{array}{l}\text { Average } \\
\text { read (bp) }\end{array}$ & $\begin{array}{l}\% \text { genome } \\
\text { sequenced }\end{array}$ & $\begin{array}{c}\text { No. Mb } \\
\text { sequenced }\end{array}$ \\
\hline Gossypioides kirkii Outgroup & 588 & 1920 & 1464 & 753 & $0.19 \%$ & 1.10 \\
\hline Gossypium raimondii D genome & 880 & 3072 & 2722 & 770 & $0.24 \%$ & 2.10 \\
\hline G. herbaceum A genome & 1667 & 6048 & 4864 & 704 & $0.21 \%$ & 3.42 \\
\hline \multirow[t]{2}{*}{ G. exiguum $\mathrm{K}$ genome } & 2460 & 10368 & 6747 & 704 & $0.19 \%$ & 4.75 \\
\hline & & & & & TOTAL & 11.4 \\
\hline
\end{tabular}

${ }^{a}$ Genome size from Wendel et al. (2002b) for G. kirkii and from Hendrix and Stewart (2005) for G. raimondii, G. herbaceum, and G. exiguum.

\section{Results}

\section{Library construction and sequence analysis}

The Gossypioides kirkii (outgroup), Gossypium raimondii (D), G. herbaceum (A), and G. exiguum (K) libraries contained 1920, 3072, 6048 , and 10,368 clones, of which 1464, 2722, 4864, and 6747 were successfully sequenced, respectively (Table 1 ). The percent of each genome sequenced $(0.19 \%-0.24 \%)$ was determined by multiplying the number of successfully sequenced clones by the average high-quality sequencing read length, divided by the estimated genome size. Sequences were queried against GenBank using BLASTX and against each other using BLASTN, and sequences were classified as described (see Methods). All types of dispersed repetitive sequences identified via this procedure were categorized into 1) gypsy-like, 2) copia-like, 3) LINE-like, 4) Mutator-like, 5) $h A T$-like, 6) $E n / S p m$-like, and 7) unknown repetitive sequences. Gypsy- and copia-like LTR retrotransposons, in addi- tion to LINE-like retroposons, were abundant in all four species (Table 2; Fig. 2). Class II DNA sequences and tandem repeats were less abundant. Some classes of dispersed repetitive sequences, such as MITEs and SINEs, were not identified in the libraries. However, because of the lack of conserved domains for these two types of sequences, they may be present in Gossypium and unidentifiable via BLAST. Copy number estimates suggest a minimum of $44 \%, 54 \%, 52 \%$, and $60 \%$ of the G. kirkii, G. raimondii, G. herbaceum, and G. exiguum genomes, respectively, are occupied by repetitive sequences alone.

Several conserved coding domains for diverse repetitive sequences were recovered from the WGS libraries when queried against Arabidopsis and Brassica databases. A total of 427 gypsylike reverse transcriptase sequences were identified. Phylogenetic analysis of 373 of these sequences confirmed the existence of three distinct classes of gypsy-like retrotransposons among the four libraries identified in the initial BLAST search (Fig. 3, and see

Repetitive Sequence Copy Number

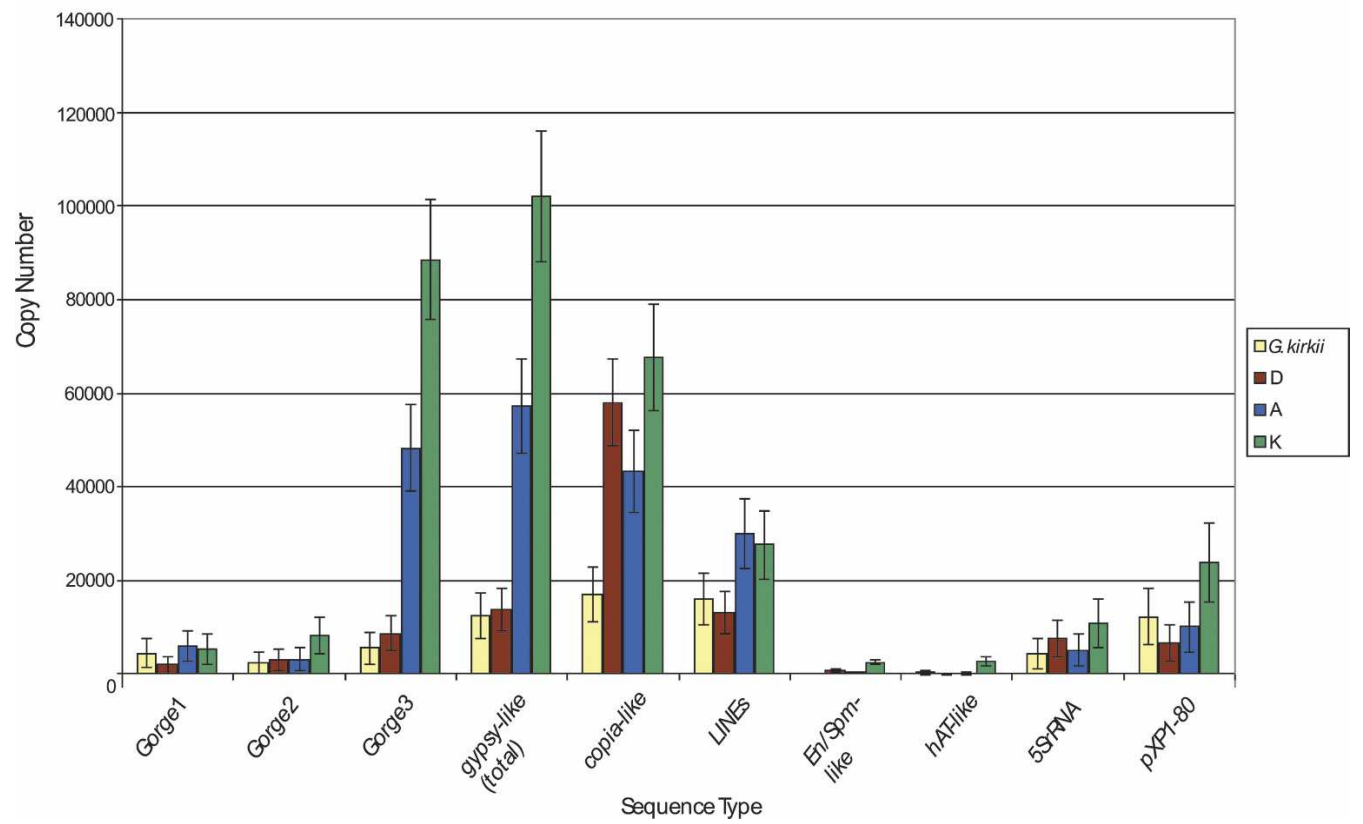

Figure 2. Copy number estimates for repetitive sequences in Gossypium. Copy numbers for repetitive sequences recovered in the WGS libraries were estimated as described (see Methods). The majority of repetitive sequences are LTR retrotransposons, particularly in the larger-genome species. In both the $\mathrm{A}$ and $\mathrm{K}$ genomes, massive amplification of Gorge3 gypsy-like sequences has occurred, contributing predominantly to genome size expansion in these two lineages. In the smallest Gossypium genome, G. raimondii (D genome), and copia-like sequences have proliferated and are primarily responsible for genome size expansion in this lineage. Class II sequences were less abundant and appear to contribute little to genome size evolution in the genus. Tandem repeats are approximately evenly distributed among all four species, with pXP1-80 sequences slightly elevated in G. exiguum (K genome). 
Table 2. Repetitive element copy number and density estimates

\begin{tabular}{|c|c|c|c|c|}
\hline & $\begin{array}{l}\text { G. kirkii Outgroup } \\
588 \mathrm{Mb}\end{array}$ & $\begin{array}{l}\text { G. raimondii } \mathrm{D} \text { genome } \\
880 \mathrm{Mb}\end{array}$ & $\begin{array}{c}\text { G. herbaceum A genome } \\
1667 \mathrm{Mb}\end{array}$ & $\begin{array}{l}\text { G. exiguum K genome } \\
2460 \mathrm{Mb}\end{array}$ \\
\hline \multicolumn{5}{|l|}{ Tandem repeats } \\
\hline 5SrRNA & $4279 \pm 3227$ & $7675 \pm 3826$ & $5073 \pm 3379$ & $10,794 \pm 5082$ \\
\hline pXP1-80 & $12,264 \pm 6098$ & $6573 \pm 3956$ & $10,101 \pm 5392$ & $23,795 \pm 8528$ \\
\hline \multicolumn{5}{|l|}{ Class II transposons } \\
\hline En/Spm-like & $\begin{array}{l}120 \pm 138 \\
\sim 0.2 \%\end{array}$ & $\begin{array}{l}835 \pm 326 \\
\sim 0.9 \%\end{array}$ & $\begin{array}{l}343 \pm 216 \\
\sim 0.2 \%\end{array}$ & $\begin{array}{c}2514 \pm 602 \\
\sim 1.0 \%\end{array}$ \\
\hline \multirow[t]{2}{*}{ hAT-like } & $305 \pm 352$ & $81 \pm 163$ & $263 \pm 304$ & $2615 \pm 986$ \\
\hline & $\sim 0.2 \%$ & $<0.1 \%$ & $<0.1 \%$ & $\sim 0.4 \%$ \\
\hline \multirow[t]{2}{*}{ Class II Total } & $3.5 \mathrm{Mb}$ & $12 \mathrm{Mb}$ & $5 \mathrm{Mb}$ & $42 \mathrm{Mb}$ \\
\hline & $<0.1 \%$ & $1.0 \%$ & $<0.1 \%$ & $\sim 1.4$ \\
\hline \multicolumn{5}{|c|}{ Class I retrotransposons } \\
\hline copia-like & $\begin{array}{c}17,006 \pm 5765 \\
9.7 \%-19.7 \%\end{array}$ & $\begin{array}{c}57,956 \pm 9300 \\
28 \%-38.7 \%\end{array}$ & $\begin{array}{c}43,181 \pm 8774 \\
10.7 \%-16.1 \%\end{array}$ & $\begin{array}{c}67,700 \pm 11,324 \\
11.7 \%-16.5 \%\end{array}$ \\
\hline \multirow[t]{2}{*}{ LINE } & $16,006 \pm 5597$ & $13,011 \pm 4502$ & $30,000 \pm 7335$ & $27,563 \pm 7271$ \\
\hline & $5.1 \%-10.6 \%$ & $2.8 \%-5.7 \%$ & $4.0 \%-6.5 \%$ & $2.4 \%-4.1 \%$ \\
\hline \multirow[t]{2}{*}{ GORGE1 gypsy-like } & $4502 \pm 2992$ & $1971 \pm 1762$ & $5909 \pm 3273$ & $5319 \pm 3205$ \\
\hline & $2.4 \%-11.9 \%$ & $0.2 \%-3.9 \%$ & $1.5 \%-5.2 \%$ & $0.8 \%-3.2 \%$ \\
\hline \multirow[t]{2}{*}{ GORGE2 gypsy-like } & $2500 \pm 2233$ & $3154 \pm 2227$ & $3181 \pm 2403$ & $8221 \pm 3983$ \\
\hline & $0.4 \%-7.5 \%$ & $1.0 \%-5.7 \%$ & $0.4 \%-3.2 \%$ & $1.6 \%-4.7 \%$ \\
\hline \multirow[t]{2}{*}{ GORGE3 gypsy-like } & $5502 \pm 3305$ & $8674 \pm 3683$ & $48,181 \pm 9257$ & $88,492 \pm 12,904$ \\
\hline & $3.5 \%-13.9 \%$ & $5.3 \%-13.0 \%$ & $22.0 \%-32.6 \%$ & $28.8 \%-38.6 \%$ \\
\hline \multirow[t]{2}{*}{ Class I Total } & $255 \mathrm{Mb}$ & $465 \mathrm{Mb}$ & $865 \mathrm{Mb}$ & $1400 \mathrm{Mb}$ \\
\hline & $42 \%$ & $53 \%$ & $52 \%$ & $58 \%$ \\
\hline
\end{tabular}

below). Reverse transcriptase sequences from copia-like retrotransposons $(n=71)$ and LINE-like retroposons $(n=20)$ in addition to transposase sequences from $h A T$-like $(n=2)$, Mutator-like $(n=1)$, and $E n / S p m$-like $(n=15)$ transposable elements were also retained for further analysis.

Tandem repeats were identified using Tandem Repeat Finder (Benson 1999). Sequences identified by TRF as tandemly repetitive were queried against GenBank using BLASTN in an attempt to assign sequence identity. Gossypium 5SrDNA sequences and a previously published Gossypium sequence, pXP1-80 (Zhao et al. 1998), were recovered from all four of the WGS libraries (see below).

\section{Copy number estimates and lineage specific amplification}

\section{Tandem repeats}

Sequences with high identity to previously described Gossypium 5SrDNA repeats were identified in all four libraries. Estimates for D- (7675 \pm 3826$)$ and A- (5073 \pm 3379$)$ genome 5SrDNA copy numbers are in agreement with previously published estimates (Cronn et al. 1996) of $4730 \pm 893$ for G. raimondii and $3415 \pm 807$ for $G$. herbaceum, those of the latter study being based on Southern hybridization data. Estimated copy numbers for 5SrDNA sequences among the four libraries fall well within the same 95\% CI (Table 2). Several other tandem repeats were recovered. One of these tandem repeats was identified as a previously published Gossypium repeat, pXP1-80 (Zhao et al. 1998). This 170- to 172-bp repeat was present in all four of the WGS libraries. Similar to the 5SrDNA repeats, copy number estimates for pXP1-80 were comparable between three of the four species (G. kirkii-12,263 \pm 6098 ; G. raimondii-6573 \pm 3956 ; $G$. herbaceum-10,101 \pm 5391 ) but elevated in $G$. exiguum $(23,795 \pm 8528)$. It may be that pXP1-80 is a centromere repeat, given that it is present in all of the WGS libraries, and its length is similar to those of published centromere repeats from Arabidopsis (178 bp), wheat (192 bp), rice (155 bp), and maize (156 bp) (Ananiev et al. 1998; Hall et al. 2003; Ito et al. 2004; Nagaki et al. 2004). Several other tandem repeats of unknown identity were identified by TRF. However, none of the remaining tandem repeats were shared among the WGS libraries, and all were present in low copy number.

\section{Class II transposons}

The three major superfamilies of Class II DNA transposons present in the WGS libraries are members of the En/Spm, Mutator, and $h A T$ DNA transposon families. Class II sequences identified were few in number, with copy number estimates suggesting that, taken as a whole, these sequences occupy $<2 \%$ of the Gossypium genome (Table 2). En/Spm-like sequences occupy $<1 \%$ of the genome in each of the four species, comprising $\sim 0.2 \%$ of the G. kirkii ( 120 copies) and G. herbaceum ( $\sim 343$ copies) genomes, but increasing in copy number in the smallest (G. raimondii$0.9 \%, \sim 835$ copies) and largest (G. exiguum-1.0\%, 2515 copies) genomes. Similarly, $h A T$-like sequences occupy $<1 \%$ of the genome in each of the four species. $h A T$-like sequence comprise only $0.2 \%$ of the $G$. kirkii ( 300 copies) genome, and an even smaller portion of the $G$. raimondii $(0.03 \%, \sim 80$ copies) and $G$. herbaceum $(0.06 \%, \sim 260$ copies) genomes. However, a large increase in copy number occurred in the $\mathrm{K}$ genome lineage $(0.4 \%$, 2600 copies). Mutator-like sequences were identified in the WGS library, but because of the large range in published lengths for these sequences and the absence of a described Mutator-like transposon for Gossypium, it was not possible to estimate their copy numbers with confidence. Additionally, because of the degenerate nature of the identified Class I sequences, it is likely that there are other undetected sequences of this type in Gossypium. There was no evidence of MITEs, TRIMs, LARDs, or Helitrons in the WGS libraries.

\section{Class I retrotransposons}

The most highly represented group of repetitive sequences within all four WGS libraries is the Class I elements (Fig. 2). Estimated total Class I copy numbers range 4.4-fold, from $45,515 \pm 9241$ in Gossypioides kirkii to $197,294 \pm 18,935$ in the $\mathrm{K}$ genome species, Gossypium exiguum. When multiplied by an 


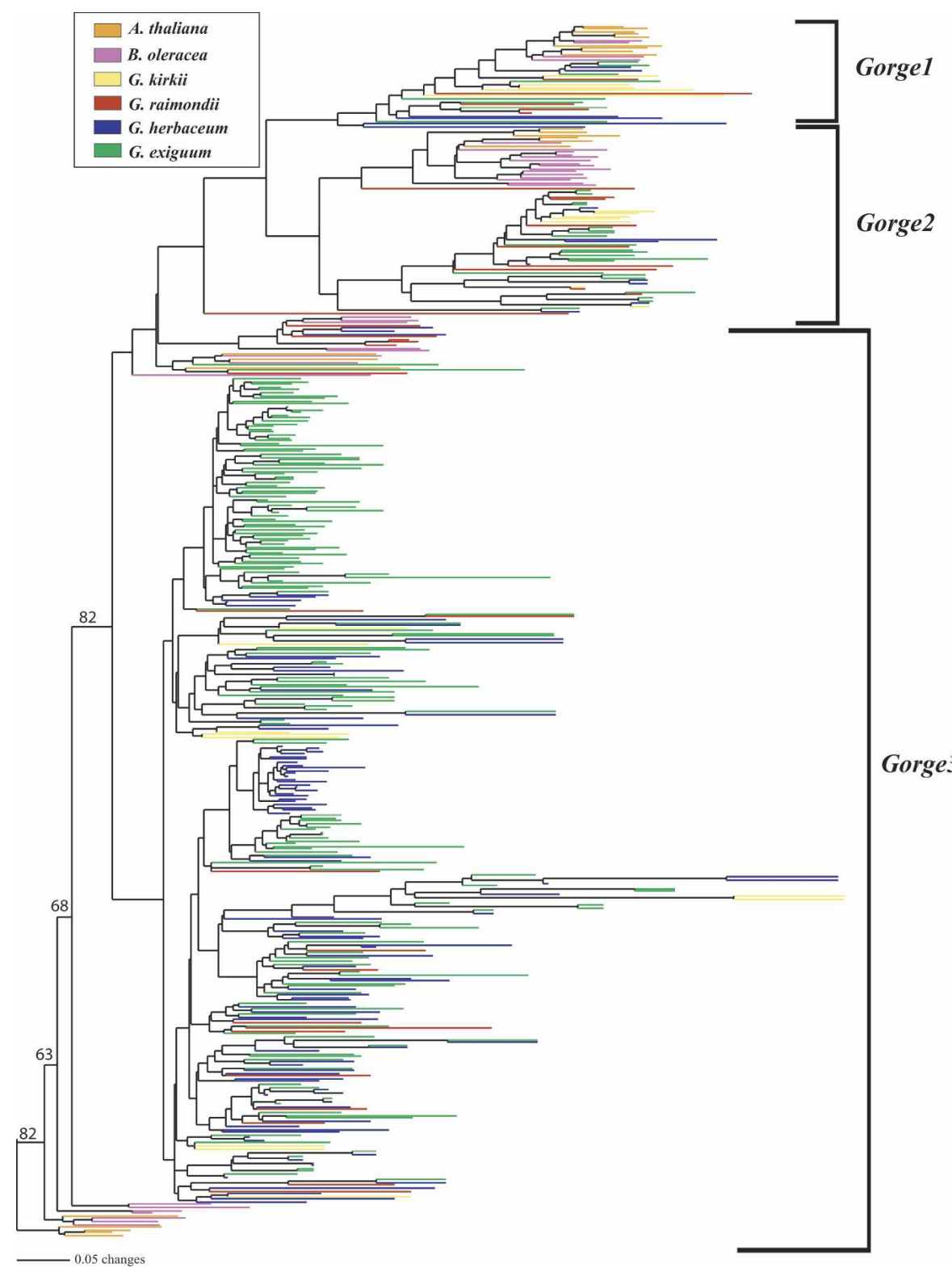

Figure 3. Neighbor-joining analysis of Gossypium gypsy-like Gorge1, 2, and 3 reverse transcriptase sequences. Unrooted Neighbor-joining analysis of 373 Gossypium, 24 Arabidopsis, and 36 Brassica gypsy reverse transcriptase sequences provides support for the three distinct classes of gypsy-like sequences in Gossypium. Gorge1 is similar to Arabidopsis gypsy sequence athila, Gorge2 is similar to maize cinful1, and Gorge3 is similar to del1-46 from Lilium henryi and dea1 from Ananas comosus. Bootstrap values for the deeper nodes are shown.

average size of $9.7 \mathrm{~kb}$ per gypsy, $5.3 \mathrm{~kb}$ per copia, and $3.5 \mathrm{~kb}$ per LINE sequence, we estimate that Class I elements occupy a minimum of $45 \%-60 \%$ of the genome for each of these species, suggesting they have amplified in each lineage approximately in proportion to genome size. However, differential proliferation among species for each group of retrotransposons is evident from the copy number estimates. Copy number estimates for copia-like retrotransposons increase proportionally with genome size, with the exception of those from the $\mathrm{D}$ genome, which are much higher than expected (Table 2). Copia-like sequences occupy $10 \%-20 \%$ of the G. kirkii, G. herbaceum (A), and G. exiguum (K) genome, but have reached considerably higher density in the species with the smallest genome size, G. raimondii (D)
(28\%-39\%). LINE-like retroposons are present in similar copy number in the D-genome $(13,011 ; 4.3 \%)$ and outgroup G. kirkii $(16,006 ; 7.9 \%)$ species, but have reached notably higher copy numbers in the A $(30,000 ; 5.3 \%)$ and $\mathrm{K}(27,563$; $3.3 \%)$ species, both of which contain much larger genomes (Table 2). We were unable to identify SINE-like retroposons (the non-autonomous counterpart of LINEs) in the WGS libraries.

The most striking example of differential lineage-specific amplification of specific groups of repetitive sequences is found among the gypsy-like sequences. BLAST analysis led to the discrimination of three different types of gypsy-like sequences present in the WGS libraries, and copy-number estimates for each of these are shown separately in Table 2 . Phylogenetic analysis of 373 gypsy-like reverse transcriptase sequences assembled from all four of the WGS libraries confirmed the existence of these three distinct classes, here designated Gorge1, Gorge2, and Gorge3, for Gossypium retrotransposon gypsy-like elements (Fig. 2). The Gorge1 group is similar to the Arabidopsis gypsy sequence athila, Gorge2 is similar to maize cinful1, and Gorge 3 is similar to del1-46 from Lilium henryi and dea1 from Ananas comosus. Copy number calculations for the three types of sequences revealed relatively stable copy numbers for Gorge 1 and Gorge 2 across all four species, although the copy number estimate for Gorge 1 in the D genome $(1971 \pm 1762)$ is somewhat lower than that of the other three species, and Gorge 2 copy number is slightly elevated in the $\mathrm{K}$ genome $(8220 \pm 3983)$ (Table 2). In contrast to this relative stability for Gorge 1 and Gorge2, there is a profound increase in copy number of Gorge 3 gypsy elements in the larger-genome species. Whereas copy numbers for Gorge 3 are similar in G. kirkii and G. raimondii $(5502 \pm 3305$ and $8674 \pm 3683$, respectively), a striking increase in copy number has taken place in both the A $(48,181 \pm 9257)$ and $\mathrm{K}(88,492 \pm 12,904)$ genome lineages. There is a sixfold increase of Gorge 3 copy number from the D to the A genome, and copy number in the A genome is nearly doubled in the $\mathrm{K}$ genome. The impact of this proliferation on genome size is apparent from density calculations: Gorge 3 occupies $\sim 9 \%$ of both the G. kirkii and D genomes, but $27.3 \%$ and $33.7 \%$ of the $\mathrm{A}$ and $\mathrm{K}$ genomes, respectively.

\section{Unidentified repetitive fraction}

Clones with no similarity to any sequence deposited in GenBank were placed in a separate database as the "unidentified fraction"

\section{Genome Research}

www.genome.org 
of each of the WGS libraries. These sequences were queried against each other using BLASTN to identify repetitive sequences that were missed during the initial BLAST search. Any sequence with $>80 \%$ sequence to at least three other clones from the same library was considered repetitive. A total of 43, 129, 364, and 603 clones from the G. kirkii, D, A, and K libraries, respectively, were considered repetitive under this criterion. The percentage of each library composed of these unidentified repetitive sequences is as follows: G. kirkii (OG)-3\%; G. raimondii (D)-5\%; G. herbaceum (A)-7.5\%; and G. exiguum (K)-9\%.

\section{Discussion}

Variation in nuclear DNA content observed within and between organisms has been a topic of interest dating back to the early 1900s, but was specifically defined and named the "C-value paradox" by Thomas in 1971 (Thomas 1971). Investigations over the past half century have revealed multiple sources of genome size variation, most commonly the differential accumulation or deletion of transposable elements. Repetitive DNA constitutes $80 \%$ of angiosperm genomes with haploid DNA content $>5.0 \mathrm{pg}$ (Flavell et al. 1974). Approximately $60 \%$ or more of the maize (SanMiguel et al. 1996, 1998; Meyers et al. 2001), wheat (Wicker et al. 2001), and barley (Vicient et al. 1999; Shirasu et al. 2000) genomes are composed of transposable elements. Nearly $25 \%$ of the maize genome consists of five classes of LTR retrotransposons alone (SanMiguel et al. 1996), and LTR retrotransposon accumulation is responsible for nearly doubling the maize genome in as little as 3 Myr (SanMiguel and Bennetzen 1998). Roughly 80\% of the wheat genome is repetitive DNA, mainly LTR retrotransposons (Kumar and Bennetzen 1999). These observations have led to an interest in the effects of repetitive DNA on genome size variation and its significance to plant fitness. Relatively little is known, however, about the evolutionary dynamics of transposable element accumulation among closely related species and how this varies among TE classes.

We constructed WGS libraries for three Gossypium and one outgroup species that range approximately fourfold in genome size in order to describe their overall genomic composition and to determine the sequences that contribute to genome size variation. Congruent with results from taxa studied to date, we found that the majority of the Gossypium genome consists of dispersed repetitive sequences. Density estimates based on previously reported repetitive sequence lengths suggest that the Gossypium genome is composed of $\sim 45 \%-60 \%$ repetitive sequences when considering only those sequences with positive BLAST matches to previously identified repetitive elements in Gossypium or in other species. This number is in agreement with estimates from other species with large genomes, such as maize, barley, and wheat (SanMiguel et al. 1996; SanMiguel and Bennetzen 1998; Kumar and Bennetzen 1999; Vicient et al. 1999; Shirasu et al. 2000; Meyers et al. 2001; Wicker et al. 2001), but differs from estimates for smaller-genome species such as Arabidopsis ( 14\%) and rice ( $26 \%)$ (The Arabidopsis Genome Initiative 2000; Jiang and Wessler 2001; Jiang et al. 2004). Additionally, given the number of repetitive sequences of unknown identity recovered in the self-BLAST searches, $45 \%-60 \%$ clearly is an underestimate of the actual repetitive fraction.

Also in agreement with results from other well-studied taxa, the majority of the identified repetitive fraction consists of Class I retrotransposon sequences. As expected based on reported esti- mates from many grasses and a few well-studied eudicots, Class II sequences were less abundant and constituted a minor fraction of the Gossypium genomes (2\%). This estimate is comparable to that from Arabidopsis (2\%-3\%) (The Arabidopsis Genome Initiative 2000), whose genome is almost five times smaller that that of $G$. kirkii (588 Mb), as well as maize (2\%) (Meyers et al. 2001), whose genome is only slightly larger than that of G. exiguum $(2460 \mathrm{Mb})$. However, this result is in contrast with that from Brassica and rice, whose genomes harbor $\sim 6 \%$ and $12 \%$ Class II DNA transposons, respectively (Jiang and Wessler 2001; Jiang et al. 2004).

\section{Lineage-specific transposition}

A key conclusion of the present study is that genome size variation in a single genus of plants reflects not only the differential amplification of diverse types of repetitive sequences, but that specific families within a repetitive sequence type proliferate differentially as well. From a purely quantitative standpoint, much of the genome size variation observed in Gossypium is a consequence of the propagation of one particular family within the larger class of gypsy-like retrotransposons, i.e., Gorge3. Recently, a gypsy-like retrotransposon ("G45" and "G84") that is transcriptionally active was reported in the tetraploid G. barbadense (Zaki and Ghany 2004). Comparisons of this active gypsy with sequences recovered in the present study revealed a maximum of $96.1 \%$ and $80.9 \%$ amino acid sequence identity between the best BLAST hit to an A-genome Gorge3 and G45 and between Agenome Gorge3 and G84, respectively. Additionally, 165 out of 150,322 Gossypium ESTs show $>60 \%$ sequence similarity over $>75$ bp to Gorge3 (e-value cut-off of e-20, data not shown). Based on this high level of sequence identity to G45 and G84, presence in the Gossypium EST libraries, and overabundance of Gorge 3 in the WGS libraries, we believe Gorge 3 is a recently active, major constituent of the cotton genome that, like LTR retrotransposons in maize, has triggered a threefold increase in genome size over the 5-10 Myr since the diversification of the major Gossypium clades following the origin of the genus (Cronn et al. 2002).

It is interesting to note that other repetitive sequences that are less common than Gorge 3 have also been subject to lineagespecific amplification during diversification of the genus. For example, little amplification of LINE retrotransposons has occurred in the $\mathrm{D}$ genome lineage, but these sequences have proliferated in the A and K genome species. Similarly, accumulation of copialike retrotransposons has occurred in the D genome lineage, yet these repetitive elements have been suppressed in the remainder of the genus, with the proportion of the genome occupied by copias in the remaining three species being between $10 \%$ and $20 \%$. Indeed, G. raimondii is the only studied Gossypium species in which there are more copia-like than gypsy-like sequences (Fig. 2).

The most parsimonious interpretation of the copia data would invoke differential amplification in the D genome lineage. However, we cannot discount the possibility of unequal rates of DNA loss. Some species appear to be more efficient at removal of non-essential DNA, such that genome size may reflect, at least in part, differential rates of DNA loss (Petrov and Hartl 1997; Kirik et al. 2000; Petrov et al. 2000; Orel and Puchta 2003). With respect to the present study, LINE-like sequences recovered in the WGS libraries are often highly degraded and hence difficult to identify. Although the most parsimonious interpretation of our copynumber estimates is a single amplification event in the common ancestor of the $\mathrm{A}$ and $\mathrm{K}$ genome lineages, a formal alternative is that LINE-like sequences existed at an ancestrally high copy 
number and have subsequently been differentially eliminated from the species with smaller genomes (D genome and the outgroup G. kirkii).

\section{Genome size evolution in Gossypium}

At present relatively little is known about the genomic locations at which genome size evolution takes place in Gossypium. The data presented here show that specific families and classes of dispersed repetitive elements have differentially proliferated in different Gossypium lineages. Given the propensity of many highcopy-number LTR retrotransposons to accumulate in heterochromatic regions of the genome (Kumar and Bennetzen 1999), we suspect that much of the evolutionarily rapid genome size change that has arisen during the global radiation of Gossypium has occurred in these gene-poor regions. Consistent with this notion, Grover et al. (2004) investigated genome size evolution in $104 \mathrm{~kb}$ of contiguous sequence surrounding the CesA 1 gene in the Gossypium A and D genomes from tetraploid cotton. Within this genic region of the Gossypium genome, no evidence of genome size variation was apparent, suggesting that genome size evolution in Gossypium takes place in heterochromatic regions located between highly conserved, euchromatic gene islands. Evaluation of this hypothesis will require additional comparative sequence and mapping data, the latter including visualization techniques such as florescent in situ hybridization (FISH) of various transposable elements.

In addition to transposable element accumulation, other suggested mechanisms of genome size change include variation in intron length, expansion/contraction of tandem repeats, illegitimate recombination, indel bias, and unequal intrastrand homologous recombination (Petrov and Wendel 2006). Contrary to suggestions that plants with smaller genomes carry smaller introns (Deutsch and Long 1999; Vinogradov 1999), there is no apparent correlation between genome size and intron length in Gossypium (Wendel et al. 2002a; Grover et al. 2004). In fact, intron length has been shown to be highly stable across 28 orthologous sets of genes from $\mathrm{A}$ and $\mathrm{D}$ genome diploid species and the outgroup species, G. kirkii (Wendel et al. 2002a). In the present study, we find no major difference between copy numbers for tandem 5SrDNA and pXP1-80 repeats, although there is a small increase in copy number in larger genomes. However, Cronn et al. (1996) reported a 20-fold variation in 5SrDNA copy number among Gossypium species, reflecting both array expansion and contraction. Grover et al. (2004) found no evidence of an indel bias, and although there was some evidence of illegitimate recombination marked by flanking repeats of $2-15$ bp in length, the resulting deletions encompass approximately the same proportion of sequence in each genome. Similar studies from other genomic locations in Gossypium will be necessary to determine if this result is a local or global occurrence.

\section{Conclusions}

Comparative studies of genome size variation among phylogenetically characterized and closely related species serve an important role in clarifying the patterns and processes that underlie the striking genome size variation that characterizes eukaryotes in general and plants in particular. With respect to the latter, we note that the genomic architecture of most plant species remains to be elucidated, and hence mechanisms that characterize one group of plants may not be universal to, say, angiosperms in general. Our data, demonstrating that different families of differ- ent classes of TEs have differentially accumulated among closely related clades of a single plant genus, underscores what we believe will be a generality, namely, that mechanisms of genome size evolution are highly variable among even closely related lineages. Our appreciation of plant genomic architecture will continue to be enhanced as comparable studies in other plant groups accumulate. These investigations will generate a deeper understanding of the genomic landscape of different plant lineages, the scale, scope, and pace of evolutionary change responsible for the observed patterns, and insights into the mechanisms the underlie the differential accumulation of different sequence types among genomes.

\section{Methods}

\section{Construction and sequencing of WGS libraries}

WGS libraries were constructed according to Meyers et al. (2001) with minor modifications and sequenced at the Arizona Genomics Institute, University of Arizona. Briefly, total genomic DNA extracted from young leaves of a single individual was randomly sheared using a Hydroshear (Thorstenson et al. 1998) (GeneMachine), an automated hydrodynamic point-sink-based DNA shearing device (Oefner et al. 1996), at speed code 13 for 25 cycles at room temperature to obtain fragments from $G$. herbaceum (JMS), G. raimondii (JFW stock), G. exiguum (Gos 5184), and G. kirkii (JFW stock) (Fig. 1). Sheared fragments between 2500 and $6000 \mathrm{bp}$ were excised and converted to blunt-ended DNA fragments using the "End-it" DNA end repair kit (Epicentre) containing T4 DNA polymerase (for $5^{\prime} \rightarrow 3^{\prime}$ polymerase and $3^{\prime} \rightarrow 5^{\prime}$ exonuclease activities) and T4 Polynucleotide Kinase (for phosphorylation of $5^{\prime}$-ends of blunt DNA), followed by ligation into pBluescriptII KS+ (Strategene) and electroporation into Escherichia coli strain DH10B T1 phase-resistant electrocompetent cells (Invitrogen). WGS library clones were sequenced from one direction using the T7 primer (5'-TAATACGACTCACTATAGGG$3^{\prime}$ ) and BigDye Terminator v3.1 (Applied Biosystems, ABI) according to manufacturer's instruction. Cycle sequencing was performed using PTC-200 thermal cyclers (MJ Research) in a 384well format with the following regime: 35 cycles of $30 \mathrm{sec}$ at $96^{\circ} \mathrm{C}, 20 \mathrm{sec}$ at $50^{\circ} \mathrm{C}$, and $4 \mathrm{~min}$ at $60^{\circ} \mathrm{C}$. After the cyclesequencing step, the DNA was purified by ethanol precipitation.

Samples were eluted into $20 \mu \mathrm{L}$ of water and separated using ABI $3730 \times 1$ DNA sequencers $(A B I)$. Sequence data were collected and extracted using sequence analysis software (ABI). The sequencing data were base-called using the program Phred (Ewing et al. 1998). Vector and low-quality sequences were removed by the program Lucy (Chou and Holmes 2001) and then submitted to the GSS division of GenBank under accessions DX390732DX406528.

\section{Analytical framework}

The number of sequences needed to generate 95\% confidence that at least one member of a given class of sequences will be sampled was determined for each species using the following equation:

$$
\mathrm{N}_{.95}=\ln (0.05) / \ln \{1-[n(l-2 m+e) /(G-e)]\}
$$

where $\mathrm{N}_{.95}$ is the sampling effort required to be $95 \%$ confident that at least one target sequence will be sampled, $n$ is the number of targets present in the genome, $l$ is the length of the target sequence, $m$ is the estimated minimum length required to identify the sequence in a BLAST search, $e$ is the number of base pairs sequenced from each insert, and $G$ is genome size. By using this

\section{Genome Research}

www.genome.org 
equation we were able to estimate the sampling intensity needed to detect at least one repetitive sequence of an estimated length and copy number in each of the four genomes. Published data for diverse types of repetitive elements, such as Ty3-gypsy, Ty1-copia, LINE retroposons, SINEs, and MITEs of various estimated lengths $(l)$ and copy numbers $(n)$ were used to calculate $\mathrm{N}_{.95}$ in order to determine how many clones should be sequenced from each library. The value for $m$ was conservatively estimated at $m=200$ $\mathrm{bp}$, which in a BLASTX analysis would equal sequence similarity over $\geq 66$ amino acids. We estimated $e$ to be $700 \mathrm{bp}$, based on the average high-quality sequencing read length reported from the Arizona Genomics Institute. Based on these estimates, libraries were constructed that contain $1.5 \%$ (based on $\sim 5 \mathrm{~kb}$ plasmid insert length) of the genome from each species. One-pass sequencing from one end of the insert $(e \sim 700 \mathrm{bp})$ was performed, which, when totaled across the number of clones sequenced, yielded sequence data for $\sim 0.2 \%$ of each haploid genome (Table 1).

\section{Data analysis and copy number estimation}

Because of rapid sequence divergence of repetitive DNA and the limited database of repetitive sequences available in GenBank for plants closely related to Gossypium, sequences from the WGS libraries were subjected to BLASTX (amino acid) in addition to BLASTN (nucleotide) analyses at the NCBI Web site. Hits of e- 5 or better were retained for further analysis. In addition, libraries were queried against themselves in an attempt to identify families of repetitive elements not recognized in the initial search. In this self-BLAST analysis, sequences with $>80 \%$ identity over 100 bp were considered related. Clones were assigned to a general category according to their best BLAST hit. These general categories were (1) nuclear, (2) chloroplast, (3) mitochondrial, (4) repetitive, and (5) unknown.

Plant transposable elements are broadly divided into three main lineages: the "Transposons" consisting of the Class II DNA elements, the "Retrotransposons" containing the LTR Class I elements, and the "Retroposons" consisting of the non-LTR Class I elements (Eickbush and Malik 2002). Class I elements transpose via a duplicative mechanism, in which an RNA intermediate formed from the parental copy is reverse transcribed, and the newly translated copies are inserted into new positions in the genome. Class I LTR retrotransposons are subdivided into two classes, gypsy and copia-like, based on the position of the integrase coding domain. The non-LTR retroposons consist of autonomous Long Interspersed Nuclear Elements (LINEs) and the non-autonomous Small Interspersed Nuclear Elements (SINEs). Class II DNA elements transpose via a cut-and-paste mechanism in which the element is excised and inserted into a new area of the genome. DNA elements characteristically contain terminal inverted repeats (TIR) ranging from 11 to a few hundred base pairs in length, and families of elements are defined by these TIR sequences (Bennetzen 2000b). Class II transposons can be divided into three main superfamilies: $h A T$ (hobo from Drosophila, Activator of maize, and Tam from Snapdragon), Mutator, and En/ Spm, both first described in maize (Kidwell 2002). Therefore, dispersed repetitive sequences recovered from the WGS libraries were placed into the specific categories 1) gypsy-like, 2) copia-like, 3) LINE-like, 4) $h A T$-like, 5) En/Spm-like, and 6) Mutator-like. DNA sequence alignments were performed with published sequences of the same type to confirm sequence identity.

Tandem repeats were identified using the program Tandem Repeat Finder (Benson 1999). Searches were performed using the default settings. Any tandem repeat present in more than three clones with a score $>500$ was retained for further analysis. These sequences were queried against GenBank using BLASTN to search for sequence similarity to known sequences deposited in GenBank. Sequences were queried against one another to identify sequences that were shared among the libraries.

Copy numbers $(n)$ for various repetitive elements recovered from the WGS libraries were estimated according to the following equation:

$$
n=\left(X_{o b s} / N\right)(G-e)(1 /(l-2 m+e))
$$

where $X_{o b s}$ is the observed number of copies, $N$ is the total number of sequence reads, and the other variables are as before: $n=$ number of targets in the genome; $l=$ length of target sequence; $m=$ estimated minimum length required to identify sequence in a BLAST search; $e=$ number of bp sequenced from each insert; and $G=$ genome size. Published sequences for various repetitive elements were used to estimate $l$. Similar to average copia sequences in rice (5-6 kb) (McCarthy et al. 2002), an $l$ of $5.3 \mathrm{~kb}$ was used for copia-like sequences based on published data from Gossypium (Grover et al. 2004). Also in agreement with rice data for gypsy-like sequences (11-13 kb) (McCarthy et al. 2002), $l$ for gypsy-like sequences in Gossypium was set at $9.7 \mathrm{~kb}$ (C.E. Grover, unpubl.). Because no data exist for other dispersed repetitive sequences in Gossypium, the estimated lengths for the remaining repetitive sequences were established according to their closest BLAST hit from GenBank and are assigned as follows: LINE retroposon $3.5 \mathrm{~kb}$ (GenBank accession no. NP_92230 from O. sativa); En/Spm with high identity to Tam1 (Nacken et al. 1991) (GenBank accession no. X57297) $15.2 \mathrm{~kb}$; and $h A T$ with high identity to Tam3 (Hehl et al. 1991) (GenBank accession no. X55078) $3.6 \mathrm{~kb}$. Published lengths for Mutator-like sequences are highly variable (in Arabidopsis these range from 444 to $19,397 \mathrm{bp}$ ) (Yu et al. 2002); therefore, we did not attempt to estimate Mutator-like copy numbers. A recent manuscript by Rabinowicz et al. (2005) used WGS libraries to estimate gene number in various plant species. When using their data for Arabidopsis and rice in our equation, we recover comparable results to those published for these two sequenced genomes, suggesting that our equation results in reasonably accurate estimates of copy numbers.

\section{Phylogeny reconstruction}

Sequences were queried against coding domains of various repetitive sequences from Arabidopsis thaliana and Brassica oleracea obtained from S. Wessler and F. Zhang (Univ. Georgia). Amino acid sequences with an e-value of e-5 or better were imported into BioEdit (Hall 1999) and aligned using ClustalW (Johnson et al. 1994). Neighbor-Joining analysis was performed in Paup* (Swofford 2001) using the default settings.

\section{Acknowledgments}

We thank Curt Brubaker for providing Gossypium exiguum seeds and DNA; S. Wessler and F. Zhang for sharing a repetitive sequence database; Jordan Swanson for assistance with data analysis; Ryan Percifield, Corrinne Grover, and Ryan Rapp for helpful discussion; and the anonymous reviewers for their comments. This work was funded by the National Science Foundation Plant Genome program.

\section{References}

Adams, K.L. and Palmer, J.D. 2003. Evolution of mitochondrial gene content: Gene loss and transfer to the nucleus. Mol. Phylogenet. Evol. 29: 380-395. 
Ananiev, E.V., Phillips, R.L., and Rines, H.W. 1998. Chromosome-specific molecular organization of maize (Zea mays L.) centromeric regions. Proc. Natl. Acad. Sci. 95: 13073-13078.

The Arabidopsis Genome Initiative. 2000. Analysis of the genome sequence of the flowering plant Arabidopsis thaliana. Nature 408: $796-815$.

Bancroft, I. 2001. Duplicate and diverge: The evolution of plant genome microstructure. Trends Genet. 17: 89-93.

Beasley, J.O. 1941. Hybridization, cytology, and polyploidy of Gossypium. Chron. Bot. 6: 394-395.

Bennett, M.D. and Leitch, I.J. 1995. Nuclear DNA amounts in angiosperms. Ann. Bot. (Lond.) 76: 113-176.

. 1997. Nuclear DNA amount in angiosperms. Philos. Trans. R. Soc. Lond., B Biol. Sci. 334: 309-345.

. 2005. Plant genome size research: A field in focus. Ann. Bot. (Lond.) 95: 1-6.

Bennett, M.D. and Smith, J.B. 1991. Nuclear DNA amounts in angiosperms. Philos. Trans. R. Soc. Lond., B Biol. Sci. 334: 309-345.

Bennetzen, J.L. 2000a. Comparative sequence analysis of plant nuclear genomes: Microcolinearity and its many exceptions. Plant Cell 12: $1021-1029$.

. 2000b. Transposable element contributions to plant gene and genome evolution. Plant Mol. Biol. 42: 251-269.

. 2002. Mechanisms and rates of genome expansion and contraction in flowering plants. Genetica 115: 29-36.

Bennetzen, J.L. and Kellogg, E.A. 1997. Do plants have a one-way ticket to genomic obesity? Plant Cell 9: 1509-1514.

Bennetzen, J.L. and Ramakrishna, W. 2002. Numerous small rearrangements of gene content, order and orientation differentiate grass genomes. Plant Mol. Biol. 48: 821-827.

Bennetzen, J.L., Ma, J., and Devos, K.M. 2005. Mechanisms of recent genome size variation in flowering plants. Ann. Bot. (Lond.) 95: $127-132$.

Benson, G. 1999. Tandem repeats finder: A program to analyze DNA sequences. Nucleic Acids Res. 27: 573-580.

Biderre, C., Metenier, G., and Vivares, C.P. 1998. A small spliceosomal-type intron occurs in a ribosomal protein gene of the microsporidian Encephalitozoon cuniculi. Mol. Biochem. Parasitol. 74: 229-231.

Blanc, G., Barakat, A., Guyot, R., Cooke, R., and Delseny, M. 2000. Extensive duplication and reshuffling in the Arabidopsis genome. Plant Cell 12: 1093-1101.

Cavalier-Smith, T. 1985. The evolution of genome size. John Wiley, New York.

Chen, M., SanMiguel, P., and Bennetzen, J.L. 1998. Sequence organization and conservation in sh2/a1-homologous regions of sorghum and rice. Genetics 148: 435-443.

Chooi, W.Y. 1971. Variation in nuclear DNA content in the genus Vicia. Genetics 68: 195-211.

Chou, H.-H. and Holmes, M.H. 2001. DNA sequence quality trimming and vector removal. Bioinformatics 17: 1093-1104.

Cronn, R.C., Zhao, X., Paterson, A.H., and Wendel, J.F. 1996. Polymorphism and concerted evolution in a tandemly repeated gene family: 5S ribosomal DNA in diploid and allopolyploid cottons. J. Mol. Evol. 42: 685-705.

Cronn, R.C., Small, R.L., Haselkorn, T., and Wendel, J.F. 2002. Rapid diversification of the cotton genus (Gossypium: Malvaceae) revealed by analysis of sixteen nuclear and chloroplast genes. Am. J. Bot. 89: $707-725$.

Deutsch, M. and Long, M. 1999. Intron-exon structure of eukaryotic model organisms. Nucleic Acids Res. 27: 3219-3228.

Devos, K.M., Brown, J.K.M., and Bennetzen, J.L. 2002. Genome size reduction through illegitimate recombination counteracts genome expansion in Arabidopsis. Genome Res. 12: 1075-1079.

Eickbush, T.H. and Malik, H.S. 2002. Origin and evolution of retrotransposons. In Mobile DNA II (eds. N.L. Craig et al.), pp. 1111-1144. ASM Press, Washington, D.C.

Ellegren, H. 2002. Mismatch repair and mutational bias in microsatellite DNA. Trends Genet. 18: 552.

Endrizzi, J.E., Turcotte, E.L., and Kohel, R.J. 1985. Genetics, cytogenetics, and evolution of Gossypium. Adv. Genet. 23: 271-375

Ewing, B., Hillier, L., Wendl, M.C., and Green, P. 1998. Base-calling of automated sequences traces using phred. I. Accuracy assessment. Genome Res. 8: 175-185.

Filkowski, J., Kovalchuk, O., and Kovalchuk, I. 2004. Dissimilar mutation and recombination rates in Arabidopsis and tobacco. Chromosoma 166: 265-272.

Flavell, R.B., Bennett, M.D., Smith, J.B., and Smith, D.B. 1974. Genome size and the proportion of repeated nucleotide sequence DNA in plants. Biochem. Genet. 12: 257-269.

Fryxell, P.A. 1992. A revised taxonomic interpretation of Gossypium L.
(Malvaceae). Rheedea 2: 108-165.

Grant, D., Cregan, P., and Shoemaker, R.C. 2000. Genome organization in dicots: Genome duplication in Arabidopsis and synteny between soybean and Arabidopsis. Proc. Natl. Acad. Sci. 97: 4168-4173.

Gregory, T.R. 2002. A bird's eye view of the C-value enigma: Genome size, cell size, and metabolic rate in the class Aves. Evolution Int. J. Org. Evolution 56: 121-130.

. 2004. Macroevolution, hierarchy theory, and the C-value enigma. Paleobiology 30: 179-202.

Grover, C.E., Kim, H., Wing, R.A., Paterson, A.H., and Wendel, J.F. 2004 Incongruent patterns of local and global genome size evolution in cotton. Genome Res. 14: 1474-1482.

Hall, T.A. 1999. BioEdit: A user-friendly biological sequence alignment editor and analysis program for Windows 95/98/NT. Nucleic Acid Symp. Ser. 41: 95-98.

Hall, S.E., Kettler, G., and Preuss, D. 2003. Centromere satellites from Arabidopsis populations: Maintenance of conserved and variable domains. Genome Res. 13: 195-205.

Hehl, R., Nacken, W.K., Krause, A., Saedler, H., and Sommer, H. 1991. Structural analysis of Tam3, a transposable element from Antirrhinum majus, reveals homologies to the Ac element from maize. Plant Mol. Biol. 16: 369-371.

Hendrix, B. and Stewart, J.M. 2005. Estimation of the nuclear DNA content of Gossypium species. Ann. Bot. (Lond.) 95: 789-797.

Ito, H., Nasuda, S., and Endo, T.R. 2004. A direct repeat sequence associated with the centromeric retrotransposons in wheat. Genome 47: 747-756.

Jiang, N. and Wessler, S.R. 2001. Insertion preference of maize and rice miniature inverted repeat transposable elements as revealed by the analysis of nested elements. Plant Cell 13: 2533-2564.

Jiang, N., Feschotte, C., Zhang, X., and Wessler, S.R. 2004. Using rice to understand the origin and amplification of miniature inverted repeat transposable elements (MITEs). Curr. Opin. Plant Biol. 7: 115-119.

Johnson, J.D., Higgins, D.G., and Gibson, T.J. 1994. CLUSTAL W: Improving the sensitivity of progressive multiple sequence alignment through sequence weighting, position specific gap penalties and weight matrix choice. Nucleic Acids Res. 22: $4673-4680$.

Jones, R.N. and Brown, L.M. 1976. Chromosome evolution and DNA variation in Crepsis. Heredity 36: 91-104.

Kidwell, M.G. 2002. Transposable elements and the evolution of genome size in eukaryotes. Genetica 115: 49-63.

Kirik, A., Salomon, S., and Puchta, H. 2000. Species-specific double-strand break repair and genome evolution in plants. EMBO J. 2000: 5562-5566

Ku, H.-M., Vision, T., Liu, J., and Tanksley, S.D. 2000. Comparing sequenced segments of the tomato and Arabidopsis genomes: Large-scale duplication followed by selective gene loss creates a network of synteny. Proc. Natl. Acad. Sci. 97: 9121-9126.

Kumar, A. and Bennetzen, J.L. 1999. Plant retrotransposons. Annu. Rev. Genet. 33: 479-532.

Leitch, I.J., Chase, M.W., and Bennett, M.D. 1998. Phylogenetic analysis of DNA C-values provides evidence for a small ancestral genome size in flowering plants. Annals Bot. (Suppl. A) 82: 85-94.

Li, W.-H. and Graur, D. 1991. Fundamentals of molecular evolution. Sinauer Associates, Sunderland, MA.

Ma, J., Devos, K.M., and Bennetzen, J.L. 2004. Analyses of LTR-retrotransposon structures reveal recent and rapid genomic DNA loss in rice. Genome Res. 14: 860-869.

McCarthy, E.M., Liu, J., Lizhi, G., and McDonald, J.F. 2002. Long terminal repeat retrotransposons of Oryza sativa. Genome Biol. 3: $1-11$.

Meyers, B.C., Tingey, S.V., and Morgante, M. 2001. Abundance, distribution, and transcriptional activity of repetitive elements in the maize genome. Genome Res. 11: 1660-1676.

Morgante, M., Hanafey, M., and Powell, W. 2002. Microsatellites are preferentially associated with nonrepetitive DNA in plant genomes. Nat. Genet. 30: 194-200.

Nacken, W.K., Piotrowiak, R., Saedler, H., and Sommer, H. 1991. The transposable element Tam 1 from Antirrhinum majus shows structural homology to the maize transposon $\mathrm{En} / \mathrm{Spm}$ and has no sequence specificity of insertion. Mol. Genet. Genomics 228: 201-208.

Nagaki, K., Neumann, P., Zhang, D., Ouyang, S., Buell, C.R., Cheng, Z., and Jiang, J. 2004. Structure, divergence, and distribution of the CRR centromeric retrotransposon family in rice. Mol. Biol. Evol. 4: $845-855$

Oefner, P.J., Hunicke-Smith, S.P., Chiang, L., Dietrich, F., Mulligan, J., and Davis, R.W. 1996. Efficient random subcloning of DNA sheared in a recirculating point-sink flow system. Nucleic Acids Res. 24: 3879-3886.

Orel, N. and Puchta, H. 2003. Differences in the processing of DNA ends

\section{Genome Research}

www.genome.org 
in Arabidopsis thaliana and tobacco: Possible implications for genome evolution. Plant Mol. Biol. 51: 523-531.

Petrov, D.A. 2002a. DNA loss and evolution of genome size in Drosophila. Genetica 115: 81-91.

. 2002b. Mutational equilibrium model of genome size evolution. Theor. Popul. Biol. 61: 531-544.

Petrov, D.A. and Hartl, D.L. 1997. Trash DNA is what gets thrown away: High rate of DNA loss in Drosophila. Gene 205: 279-289.

Petrov, D.A. and Wendel, J.F. 2006. Genome evolution in eukaryotes: The genome size perspective. In Evolutionary genetics: Concepts and case studies (eds. C.W. Fox and J.B. Wolf), pp. 144-156. Oxford University Press, Oxford, UK.

Petrov, D.A., Sangster, T.A., Johnston, J.S., Hartl, D.L., and Shaw, K.L. 2000. Evidence for DNA loss as a deteminant of genome size. Science 287: $1060-1062$.

Price, H.J. 1988. Nuclear DNA content variation within angiosperm species. Evol. Trends Plants 2: 53-60.

Rabinowicz, P.D., Citek, R., Budiman, M.A., Nunberg, A., Bedell, J.A., Lakey, N., O'Shaughnessy, A.L., Nascimento, L.U., McCombie, W.R., and Martienssen, R.A. 2005. Differential methylation of genes and repeats in land plants. Genome Res. 15: 1431-1440.

SanMiguel, P. and Bennetzen, J.L. 1998. Evidence that a recent increase in maize genome size was caused by the massive amplification of intergene retrotransposons. Ann. Bot. (Lond.) 82: 37-44.

SanMiguel, P., Tikhonov, A., Jin, Y.K., Motchoulskaia, N., Zakharov, D., Melake-Berhan, A., Springer, P.S., Edwards, K.J., Lee, M., Avramova, Z., et al. 1996. Nested retrotransposons in the intergenic regions of the maize genome. Science 274: 765-768.

SanMiguel, P., Gaut, B.S., Tikhonov, A., Nakajima, Y., and Bennetzen, J.L. 1998. The paleontology of intergene retrotransposons of maize. Nat. Genet. 20: 43-45.

Seelanan, T., Schnabel, A., and Wendel, J.F. 1997. Congruence and consensus in the cotton tribe. Syst. Bot. 22: 259-290.

Shahmuradov, I.A., Akbarova, Y.Y., Solovyev, V.V., and Aliyev, J.A. 2003. Abundance of plastid DNA insertions in nuclear genomes of rice and Arabidopsis. Plant Mol. Biol. 52: 923-934.

Shepherd, N.S., Schwarz-Sommer, Z., Blumberg vel Spalve, J., Gupta, M., Wienand, U., and Saidler, H. 1984. Similarity of the Cin1 repetitive family of Zea mays to eukaryotic transposable elements. Nature 307: 185-187.

Shirasu, K., Schulman, A.H., Lahaye, T., and Schulze-Lefert, P. 2000. A contiguous 66-kb barley DNA sequence provides evidence for reversible genome expansion. Genome Res. 10: 908-915.

Small, R.L., Ryburn, J.A., Cronn, R.C., Seelanan, T., and Wendel, J.F. 1998. The tortoise and the hare: Choosing between noncoding plastome and nuclear Adh sequences for phylogeny reconstruction in a recently diverged plant group. Am. J. Bot. 85: 1301-1315.

Small, R.L., Ryburn, J.A., and Wendel, J.F. 1999. Low levels of nucleotide diversity at homoeologous Adh loci in allotetraploid cotton (Gossypium L.). Mol. Biol. Evol. 16: 491-501.

Sparrow, A.H., Price, H.J., and Underbrink, A.G. 1972. A survey of DNA content per cell and per chromosome of prokaryotic and eukaryotic organisms: Some evolutionary considerations. Brookhaven Symp. Biol. 23: 451-494.

Swofford, D.L. 2001. PAUP*. Phylogenetic analysis using parsimony (*and other methods). Version 4. Sinauer Associates, Sunderland, MA.

Thomas, C.A. 1971. The genetic organisation of chromosomes. Annu. Rev. Genet. 5: 237-256.

Thorstenson, T.R., Hunicke-Smith, S.P., Oefner, P.J., and Davis, R.W. 1998. An automated hydrodynamic process for controlled, unbiased DNA shearing. Genome Res. 8: 848-855.

Tikhonov, A., SanMiguel, P., Nakajima, Y., Gorenstein, N.M., Bennetzen, J.L., and Avramova, Z. 1999. Colinearity and its exceptions in orthologous adh regions of maize and sorghum. Proc. Natl. Acad. Sci. 96: 7409-7414

Vicient, C.M., Suoniemi, A., Anamthawat-Jonsson, K., Tanskanen, J., Beharav, A., Nevo, E., and Schulman, A.H. 1999. Retrotransposon BARE-1 and its role in genome evolution in the genus Hordeum. Plant Cell 11: 1769-1784.

Vinogradov, A.E. 1999. Intron-genome size relationship on a large evolutionary scale. J. Mol. Evol. 49: 376-384.

Vision, T.J., Brown, D.G., and Tanksley, S.D. 2000. The origins of genomic duplications in Arabidopsis. Science 290: 2114-2117.

Wendel, J.F. 2000. Genome evolution in polyploids. Plant Mol. Biol. 42: 225-249.

Wendel, J.F. and Albert, V.A. 1992. Pylogenetics of the cotton genus (Gossypium): Character-state weighted parsimony analysis of chloroplast-DNA restriction site data and its systematic and biogeographic implications. Syst. Bot. 17: 115-143.

Wendel, J.F. and Cronn, R.C. 2003. Polyploidy and the evolutionary history of cotton. Adv. Agron. 78: 139-186.

Wendel, J.F., Cronn, R.C., Alvarez, I., Liu, B., Small, R.L., and Senchina, D.S. 2002a. Intron size and genome size in plants. Mol. Biol. Evol. 19: 2346-2352.

Wendel, J.F., Cronn, R.C., Johnston, J.S., and Price, H.J. 2002b. Feast and famine in plant genomes. Genetica 115: $37-47$.

Wicker, T., Stein, N., Albar, L., Feuillet, C., Schlagenhauf, E., and Keller, B. 2001. Analysis of a contiguous $211 \mathrm{~kb}$ sequence in diploid wheat (Triticum monococcum L.) reveals multiple mechanisms of genome evolution. Plant J. 26: 307-316.

Wicker, T., Yahiaoui, N., Guyot, R., Schlagenhauf, E., Liu, Z.-D., Dubcovsky, J., and Keller, B. 2003. Rapid genome divergence at orthologous low molecular weight glutenin loci of the $\mathrm{A}$ and $\mathrm{A}^{\mathrm{m}}$ genomes of wheat. Plant Cell 15: 1186-1197.

Yu, J., Hu, S., Wang, J., Wong, G.K., Li, S., Liu, B., Deng, Y., Dai, L., Zhou, Y., Zhang, X., et al. 2002. A draft sequence of the rice genome (Oryza sativa L. ssp. indica). Science 296: 79-92.

Zaki, E.A. and Ghany, A.A.A. 2004. Ty3/gypsy retro-transposons in Egyptian cotton (G. barbadense). J. Cotton. Sci. 8: 179-185.

Zhang, J. 2003. Evolution by gene duplication: An update. Trends Ecol. Evol. 18: 292-298.

Zhao, X.P., Si, Y., Hanson, R.E., Crane, C.F., Price, H.J., Stelly, D.M., Wendel, J.F., and Paterson, A.H. 1998. Dispersed repetitive DNA has spread to new genomes since polyploid formation in cotton. Genome Res. 8: 479-492.

Received March 3, 2006; accepted in revised form May 22, 2006. 


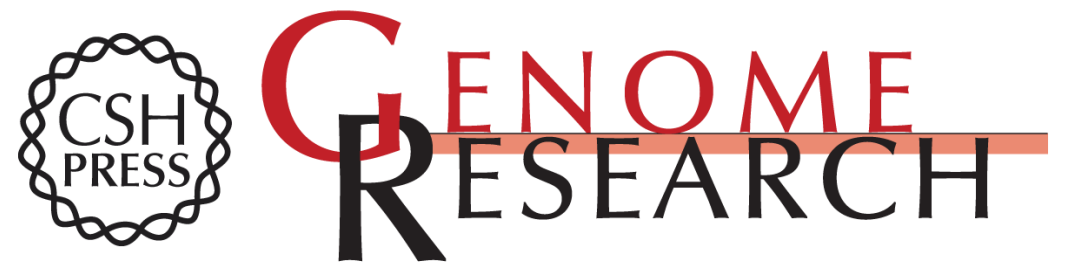

\section{Differential lineage-specific amplification of transposable elements is responsible for genome size variation in Gossypium}

Jennifer S. Hawkins, HyeRan Kim, John D. Nason, et al.

Genome Res. 2006 16: 1252-1261

Access the most recent version at doi:10.1101/gr.5282906

References This article cites 89 articles, 23 of which can be accessed free at:

http://genome.cshlp.org/content/16/10/1252.full.html\#ref-list-1

\section{License}

Email Alerting Receive free email alerts when new articles cite this article - sign up in the box at the Service top right corner of the article or click here.

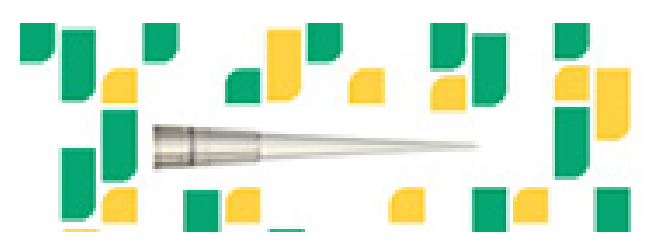

To subscribe to Genome Research go to: https://genome.cshlp.org/subscriptions 